\title{
Association between alcohol use and sexually transmitted infection incidence among Kenyan women engaged in transactional sex
}

\author{
Kate S. Wilson ${ }^{\mathrm{a}}$, Katherine Odem-Davis ${ }^{\mathrm{d}}$, Juma Shafi ${ }^{\mathrm{e}}$, Francis Kashonga $^{\mathrm{e}}$, George Wanje ${ }^{\mathrm{e}}$, \\ Linnet Masese $^{a}$, Kishor Mandaliya ${ }^{\dagger}$, Walter Jaoko $^{e}$, and R. Scott Mcclelland ${ }^{a, b, c, e}$ \\ aDepartment of Epidemiology, University of Washington, Seattle, USA \\ ${ }^{b}$ Department of Medicine, University of Washington, Seattle, USA \\ 'Department of Global Health, University of Washington, Seattle, USA \\ dFred Hutchison Cancer Research Center, Seattle, USA \\ eUniversity of Nairobi, Nairobi, Kenya \\ ${ }^{f}$ Coast Province General Hospital, Mombasa, Kenya
}

\begin{abstract}
Few prospective studies have evaluated the association between alcohol use and STI acquisition among African women. We examined the association between baseline drinking frequency and STIs in a cohort of Kenyan women reporting transactional sex. The association between alcohol use and STI differed significantly by HIV status. Among 139 HIV-positive women, STI acquisition was significantly associated with consuming 1-7 drinks/week and marginally associated with $\geq 8$ drinks/week in unadjusted analyses. However, no association between alcohol use and STIs was observed among 335 HIV-negative women. Addressing alcohol use within comprehensive HIV care may also reduce the burden of STIs among high-risk women.
\end{abstract}

\author{
Keywords \\ alcohol use; STI; African women; prospective
}

\section{Introduction}

Sexually transmitted infections (STI) contribute substantial health burdens to women in Africa, including increased risk of HIV acquisition and ongoing transmission (1). Alcohol use in Africa is common and has been implicated as a potentially important risk factor for HIV and other STIs (2-4). Several African studies have shown that the frequency and pattern of alcohol use are associated with prevalent STIs (5), sexual risk behaviors (6) and positive HIV status (7). Alcohol use may increase the risk of STI acquisition by increasing risky

Corresponding Author: Kate Wilson, Department of Epidemiology, University of Washington, 325 Ninth Ave, Box 359909, Seattle, WA 98104 Phone \# 206-543-4278, Fax \# 206-543-4818, ksw@uw.edu. 
sexual behavior (2). It is also notable that alcohol consumption may be more frequent among women engaged in sex work compared to the general population (8). Accordingly, alcohol use may be a particularly important risk factor for STIs among sex workers. These women may have higher risk partners, solicit clients in bars, and drink alcohol with potential sex partners, compromising their ability to negotiate safer sex (8).

Most studies evaluating the association between alcohol use and STIs in African women have been cross-sectional $(2,3)$. As a result, these studies have not been able to examine temporal relationships between alcohol use and STI risk. The prospective studies that are available provide some insight into the association between alcohol use and STI acquisition, but have limitations including the use of self-reported sexual risk behaviors (6), self-reported STIs (2), or only HIV acquisition as endpoints (4). One prospective analysis of data from HIV-negative sex workers in Kenya found that daily alcohol use was an independent risk factor for laboratory-confirmed curable STIs (9). Additional studies in both HIV-positive and HIV-negative African women will be important to further understand the nature of the association between alcohol use and STI risk, and to inform more comprehensive STI prevention strategies. It is important to include HIV-positive women in these studies because HIV-positive women may have a higher risk of STI acquisition compared to HIV-negative women due to greater biological susceptibility as well as more exposure to sexual networks with higher STI prevalence $(1,2)$.

In the present study, we hypothesized that alcohol use would be associated with increased risk of STIs among African women reporting transactional sex. We evaluated the association between self-reported baseline alcohol use and incidence of laboratory-diagnosed curable STIs using data from a cohort of HIV-positive and HIV-negative Kenyan women.

\section{Methods}

\section{Participants and procedures}

We conducted a prospective longitudinal analysis using data from the Mombasa Cohort, a prospective cohort study of risk factors for HIV acquisition, transmission, and disease progression among women engaged in transactional sex in Mombasa, Kenya. The cohort was initiated in 1993 and detailed procedures have been published (10). Women were eligible to join the cohort if they were $18-50$ years old, reported engaging in sex for cash or in-kind payment, and were willing and able to provide informed consent. At enrollment and monthly follow-up visits, participants completed a standardized fact-to-face interview with a trained study nurse to collect information about their demographic characteristics, medical histories, and sexual behaviors. Women were asked about sexual behavior in the past week at each follow-up visit in order to estimate the proportion of unprotected sex. The questions were: "During the past week: How many times did you have sexual intercourse? How many times did you have sexual intercourse with condoms?"

A study physician conducted a physical examination including a speculum-assisted pelvic examination with collection of vaginal and cervical specimens for laboratory diagnosis of STIs. Participants received free outpatient medical care, including treatment for STIs, according to World Health Organization guidelines. Women who reported symptoms of a 
genital tract infection were treated syndromically at the same visit and were given a followup appointment one week later. At that time, they were provided with additional treatment if STIs were identified by laboratory testing that were not covered with the medications provided at the prior visit. Women who were HIV-positive at enrollment or who seroconverted during follow-up were offered free comprehensive HIV care, including antiretroviral therapy (ART), according to Kenyan national treatment guidelines. After April 2008, HIV-positive women who were not taking ART were asked to return every three months with additional visits as needed based on their symptoms. This decrease from monthly to quarterly visits aligned follow-up in the research cohort with local clinical standards for HIV-positive individuals not taking ART.

At enrollment, women were asked about the number of alcoholic drinks they consumed in an average week. The question was: "Do you drink alcohol?" If a woman said yes, she was asked: "How many drinks per week?" They were also asked about their use of other drugs (marijuana, miraa, cocaine, and IV drugs). Nucleic acid amplification testing (NAAT) for Neisseria gonorrhoeae and Chlamydia trachomatis was conducted using the Gen-Probe APTIMA Combo 2 assay (Gen Probe, San Diego, CA). In addition, culture for $N$. gonorrhoeae was performed on modified Thayer-Martin media. Trichomonas vaginalis was diagnosed by microscopic examination of a vaginal saline wet preparation for motile parasites. HIV-1 sero-status was determined by enzyme-linked immunosorbent assay (Detect HIV 1/2 [BioChem Immunosystems, Montreal Canada]) or PT-HIV 1, 2-96 [Pishtaz Teb Diagnostics, Tehran, Iran]. Positive test results were confirmed using a second enzymelinked immunosorbent assay (Recombigen [Cambridge Biotech, Worchester, MA]).

\section{Data Analysis}

Women who were enrolled in the cohort between August 2006 (when Gen-Probe $N$. gonorrhoeae and C. trachomatis testing was initiated in the cohort) and December 2011 and had at least one visit with a NAAT result were included in the analysis. Four women with missing or inconclusive HIV status were excluded. Because alcohol use can change over time, we included no more than two years of follow-up per woman. The primary exposure was baseline alcohol use (drinks per week), which was categorized as no drinks, 1-7, or $\geq 8$ drinks/week. These categories were chosen based on the distribution of the data and our prior studies in the same cohort (10). The binary outcome, measured at each follow-up visit, was a combined endpoint indicating a positive test for any one or more STIs $(N$. gonorrohoeae and $C$. trachomatis, . vaginalis) compared to no STI. The association between each level of alcohol use and STIs was evaluated with logistic regression using generalized estimating equations (GEE) with robust standard errors and exchangeable working correlation structure to estimate odds ratios (OR) and 95\% confidence intervals (CI) based on a Wald test statistic. This method accounted for correlation between multiple visits for the same woman. Additional NAAT testing for $N$. gonorrohoeae and $C$. trachomatis was performed from August 2006 through April 2008 and then quarterly thereafter. Because our hypothesis focused on STI outcomes only those visits with NAAT results were included in the analysis. 
Pre-specified potential confounding factors were included in all adjusted models and included age (continuous linear) and work place (bar, nightclub, home/other location). Additional potential confounding factors including years of education (continuous linear), religion (Protestant, Catholic, Muslim, other), pregnancy at enrollment, marital status (never, currently married, divorced/widowed), marijuana use (binary), and hormonal contraceptive use (binary) were considered if they were independently associated with the STI outcome in univariate analysis $(\alpha=0.1)$. Women were classified as having unprotected sex at a follow-up visit when their total reported number of sex acts exceeded the number of reported sex acts with a condom. In light of data from prior studies suggesting an association between alcohol use and HIV acquisition (3), we tested an a priori hypothesis that the association between alcohol use and STI might differ in HIV-positive versus HIV-negative women. In addition, we expected that the association between alcohol use and STI might differ by HIV-status because of evidence that HIV is associated with increased risk of STIs (5). This analysis was performed by including an interaction term for HIV status and baseline alcohol use in the model. We also conducted an analysis to examine whether the association between alcohol use and STI could be explained by reported unprotected sex at follow-up visit. We compared women with at least one return visit with a NAAT result to women who never had NAAT using Mann-Whitney (MW) test of medians for continuous variables and Pearson's chi-square tests for categorical variables.

\section{Results}

Overall, 622 women were enrolled between August 2006 and December 2011. Of these, 474 (76.2\%) had at least one return visit in which NAAT testing for $N$. gonorrhoeae and $C$. trachomatis was performed. The final sample of 474 women contributed 1,915 visits to the analyses. The median number of follow-up visits was 6.5 (interquartile range [IQR] 3.5, 10).

Participants in this study had a median age of 29 years (IQR 25, 35). Most women were divorced or widowed $(270,57.0 \%)$. Over half of the women worked in bars $(268,56.6 \%)$, while the remainder worked in nightclubs $(113,24.0 \%)$ or at home/other locations $(93$, $19.6 \%$ ). The women reported a median of two (IRQ 0,3) years in sex work. Of $165(34.1 \%)$ women who reported using a modern contraceptive method besides condoms, 102 (61.8\%) were using injectable hormonal contraception. Only 11 women (2.3\% of 474) reported current pregnancy. At enrollment, 139 (29.3\%) women were HIV-positive. Four women sero-converted during follow-up. Of the HIV-positive women, 5 (3.6\%) were on ART at enrollment and $26(18.7 \%)$ initiated ART during follow-up.

Among 349 (73.6\%) women reporting any alcohol consumption, 267 (56.3\%) reported having 1-7 drinks per week and $82(17.3 \%)$ reported $\geq 8$ drinks/week. Only $16(3.4 \%)$ women reported marijuana use, $2(0.3 \%)$ reported cocaine use, and none reported injection drug use. At enrollment, the median number of sex partners reported in the past week was one (IQR 1,3) and median sex acts was two (IQR 1,3). Over one quarter of the women (129, $27.2 \%$ ) reported at least one act of unprotected sex in the past week.

Thirty nine (8.2\%) women had an STI at enrollment. Of those, 20 (4.2\% of 473) tested positive for $T$. vaginalis and 8 (1.7\% of 471) tested positive for $N$. gonorrhoeae. Among a 
subset of 223 women who received NAAT testing at enrollment, 10 (4.5\% of 223) tested positive for $N$. gonorrhoeae and 9 (4.0\% of 223) tested positive for C. trachomatis.

We compared the 474 women included in this analysis to the 148 women who were excluded because they had no return visits with NAAT testing. The women who were excluded were younger (median $26[23,31]$ versus $29[25,35]$ years, MW $\chi^{2}=19.77$, $\mathrm{p}<0.001)$, and were more likely to be HIV negative $\left(124,85.0 \%\right.$ versus $335,70.7 \% ; \chi^{2}=$ 7.62, $\mathrm{p}<0.01$ ), work in nightclubs $\left(70,47.6 \%\right.$ versus $\left.114,24.1 \% ; \chi^{2}=30.59 \mathrm{p}<0.001\right)$, report alcohol use $\left(117,79.6 \%\right.$ versus $349,73.6 \% ; \chi^{2}=17.38, p<0.001$ and marijuana use $(13$, $8.9 \%$ versus $\left.16,3.4 \% ; \chi^{2}=7.54 \mathrm{p}<0.01\right)$. In addition, the excluded women reported more sex partners (median $2[1,3]$ versus $1[1,3]$; MW $\chi^{2}=4.52, \mathrm{p}<0.05$ ) and more sex acts (median $2[1,4]$ versus $2[1,3]$; MW $\chi^{2}=6.59, \mathrm{p}=0.013$ ) but did not differ from the included women by reported frequency of unprotected sex $\left(38,26.0 \%\right.$ versus $129,27.2 \% ; \chi^{2}=0.11$, $\mathrm{p}=0.76)$.

\section{Association between alcohol use and STI acquisition}

Overall, there was no statistically significant association between alcohol use and incident STIs (1-7 drinks/week OR 1.5 [95\% CI: 0.9, 2.5], $\mathrm{p}=0.13 ; \geq 8$ drinks/week OR 1.4 [95\% CI: 0.6, 3.1], $\mathrm{p}=0.40$; Wald test $=2.35$, $\mathrm{p}=0.31$ ). However, the association between alcohol use and STI risk was significantly different in HIV-positive compared to HIV-negative women ( $\mathrm{p}=0.05$ ), so the remainder of the results are presented stratified by HIV status (Table I). Although levels of alcohol use were similar between HIV-positive and HIVnegative women, the frequency of STIs at follow-up visits was higher among HIV-positive women. Compared to HIV-positive visits among women with no reported alcohol use at baseline, there was a significantly increased risk of STI at HIV-positive visits among women who reported 1-7 drinks/week (OR 2.3 [95\% CI: 1.0, 5.5], $\mathrm{p}=0.05$ ). There was also a trend suggesting increased risk of STIs associated with $>8$ drinks/week (OR 3.2 [95\% CI: 1.0, 10.6], $\mathrm{p}=0.06$; Wald test $=4.85, \mathrm{p}=0.09$ ). However, this was not statistically significant at the $a=0.05$ level. These results were similar after adjusting for age, marijuana use, and place of work, although these associations were no longer statistically significant at $\alpha=0.05$ level. Adjustment for unprotected sex reported in the past week did not substantially change the multivariate results. In contrast to the observed association in HIV-positive women, there was no evidence of an association between alcohol use and STI acquisition in HIV-negative women (unadjusted OR 1.0 [95\% CI: 0.5, 1.9], p=1.0), and $\geq 8$ drinks/week (OR 0.7 [95\% CI: $0.3,1.9], \mathrm{p}=0.5$; Wald test $=0.5, \mathrm{p}=0.78$ ).

\section{Discussion}

In this prospective analysis of data from a cohort of high-risk Kenyan women, alcohol use was associated with an approximately two-fold increased risk of STI acquisition in HIVpositive participants that was marginally significant. After adjustment for age, workplace, and marijuana use, the association between 1-7 drinks per week compared to no drinks and STI risk remained marginally significant while the association between 8 or more drinks per week and STI risk became non-significant. In contrast, alcohol use was not associated with increased STI risk in women who were HIV-negative. This prospective study adds to the 
limited literature on the relationship between alcohol use and STI acquisition in African settings.

The results of the present analysis are generally consistent with findings from crosssectional studies that have found a significant association between alcohol use and prevalent STIs among African women $(2,8)$. One previous prospective study in African women, conducted among HIV-negative Kenyan sex workers found that daily alcohol use was associated with a 1.4-fold increase in the risk of acquiring curable STIs (9).

We did not observe a significant association between alcohol use and STI risk in HIV negative women. This may have been due to inadequate power, given the modest sample size in the study and a lower number of STIs among HIV-negative women compared to HIV-positive women. However, there are other possible explanations. For example, HIVpositive women may have participated in overall riskier sexual networks with more STIinfected members compared to their HIV-negative peers (8). In addition, there is some evidence that HIV-positive women have greater biological susceptibility to some STIs compared to HIV-negative women (1), which could help to explain the observed difference in the association between alcohol use and STIs in these groups.

One plausible explanation for the observed association between alcohol use and increased STI risk is that alcohol use leads to riskier sexual behaviors (3). While we were unable to show that the association between alcohol use and STIs in HIV-positive women was mediated through higher rates of unprotected sex, it is important to note that we only measured risk behavior during the past week. In contrast, STIs could have occurred at any time between follow-up visits. There may also have been underreporting of the frequency of unprotected sex.

This study had several strengths. The longitudinal design enabled us to assess the temporal relationship between reported alcohol use and STI acquisition. Our sample included both HIV-positive and HIV-negative women, allowing us to compare these groups. Furthermore, we used laboratory-confirmed STI endpoints, including NAAT, which has high sensitivity and specificity for detecting $N$. gonorrhoeae and C. trachomatis (11).

This study also had limitations. First, because we measured alcohol use by self-report, there may have been underreporting due to social desirability bias. Second, we used a single global measure of alcohol use frequency in drinks per week. Measuring patterns of alcohol use, alcohol use disorders, and event level measures (i.e. alcohol use at the time of sexual activity) can yield more precise estimates of the effects of alcohol on health outcomes $(2,3)$. Third, alcohol use was only measured at baseline and may have varied during follow-up. Future research with time-varying measures of alcohol use will be important to further characterize the relationship between alcohol and STI risk among HIV-positive and HIVnegative women. Fourth, about $24 \%$ of women were excluded from the analysis because they did not have visits with NAAT results, in most cases because they did not return for follow-up visits. This may limit the generalizability. Fifth, our results showing effect modification and an association between alcohol use and higher STIs among HIV-positive women were of borderline statistical significance. These results should be interpreted with 
caution and evaluated in future studies with HIV-positive and HIV-negative women. Finally, our study included African women who reported engaging in transactional sex, so these results may not reflect the relationship between alcohol use and risk of STI acquisition in the general population. Nonetheless, women involved in sex work are an important population that contributes to HIV transmission even in countries with mature epidemics (12). Prioritizing STI prevention in this marginalized population could help to reduce HIV transmission.

\section{Conclusions}

There is growing consensus that alcohol use is an important modifiable risk factor that should be addressed within HIV care programs in Africa $(2,3,8)$. Promising interventions that address alcohol use problems as a component of comprehensive HIV prevention and care could also reduce the burden of curable STIs among high-risk African women.

\section{Acknowledgments}

We are grateful to the study participants and our research, clinical, laboratory, outreach, and administrative staff for making this study possible. We would also like to acknowledge Mariana Rios for the Spanish translation of the abstract. This study was funded by a grant from the National Institutes of Health (R01 HD072617). K.S.W. was supported by the University of Washington Center for STD and AIDS (grant T32 AI07140). Infrastructure support for the Mombasa research site was provided by the University of Washington's Center for AIDS Research (CFAR), an NIH funded program (P30 AI027757) which is supported by the following centers: NIAID, NCI, NIMH, NIDA, NICHD, NHLBI, NCCAM.

\section{References}

1. Johnson LF, Lewis DA. The effect of genital tract infections on HIV-1 shedding in the genital tract: a systematic review and meta-analysis. Sex Transm Dis. 2008; 35(11):946-59. [PubMed: 18685546]

2. Woolf-King SE, Maisto SA. Alcohol use and high-risk sexual behavior in Sub-Saharan Africa: a narrative review. Arch Sex Behav. 2011; 40(1):17-42. [PubMed: 19705274]

3. Kalichman SC, Simbayi LC, Kaufman M, Cain D, Jooste S. Alcohol use and sexual risks for HIV/ AIDS in sub-Saharan Africa: systematic review of empirical findings. Prev Sci. 2007; 8(2):141-51. [PubMed: 17265194]

4. Baliunas D, Rehm J, Irving H, Shuper P. Alcohol consumption and risk of incident human immunodeficiency virus infection: a meta-analysis. Int J Public Health. 2010; 55(3):159-66. [PubMed: 19949966]

5. Kalichman SC, Pellowski J, Turner C. Prevalence of sexually transmitted co-infections in people living with HIV/AIDS: systematic review with implications for using HIV treatments for prevention. Sex Transm Inf. 2011; 87(3):183-90.

6. Shuper PA, Joharchi N, Irving H, Rehm J. Alcohol as a correlate of unprotected sexual behavior among people living with HIV/AIDS: review and meta-analysis. AIDS Behav. 2009; 13(6):102136. [PubMed: 19618261]

7. Fisher JC, Bang H, Kapiga SH. The association between HIV infection and alcohol use: a systematic review and meta-analysis of African studies. Sex Transm Dis. 2007; 34(11):856-63. [PubMed: 18049422]

8. Chersich MF, Luchters SM, Malonza IM, Mwarogo P, King'ola N, Temmerman M. Heavy episodic drinking among Kenyan female sex workers is associated with unsafe sex, sexual violence and sexually transmitted infections. Int J STD \& AIDS. 2007; 18(11):764-9. [PubMed: 18005511]

9. Yadav G, Saskin R, Ngugi E, Kimani J, Keli F, Fonck K, et al. Associations of sexual risk taking among Kenyan female sex workers after enrollment in an HIV-1 prevention trial. J Acquir Immune Defic Syndr. 2005; 38(3):329-34. [PubMed: 15735453] 
10. McClelland RS, Lavreys L, Katingima C, Overbaugh J, Chohan V, Mandaliya K, et al. Contribution of HIV-1 infection to acquisition of sexually transmitted disease: a 10-year prospective study. J Inf Dis. 2005; 191:333-8. [PubMed: 15633091]

11. Gaydos CA, Quinn TC, Willis D, Weissfeld A, Hook EW, Martin DH, et al. Performance of the APTIMA Combo 2 assay for detection of Chlamydia trachomatis and Neisseria gonorrhoeae in female urine and endocervical swab specimens. J Clin Microbiol. 2003; 41(1):304-9. [PubMed: 12517865]

12. Chen L, Jha P, Stirling B, Sgaier SK, Daid T, Kaul R, et al. Sexual risk factors for HIV infection in early and advanced HIV epidemics in sub-Saharan Africa: systematic overview of 68 epidemiological studies. PLoS One. 2007; 2(10):e1001. [PubMed: 17912340] 


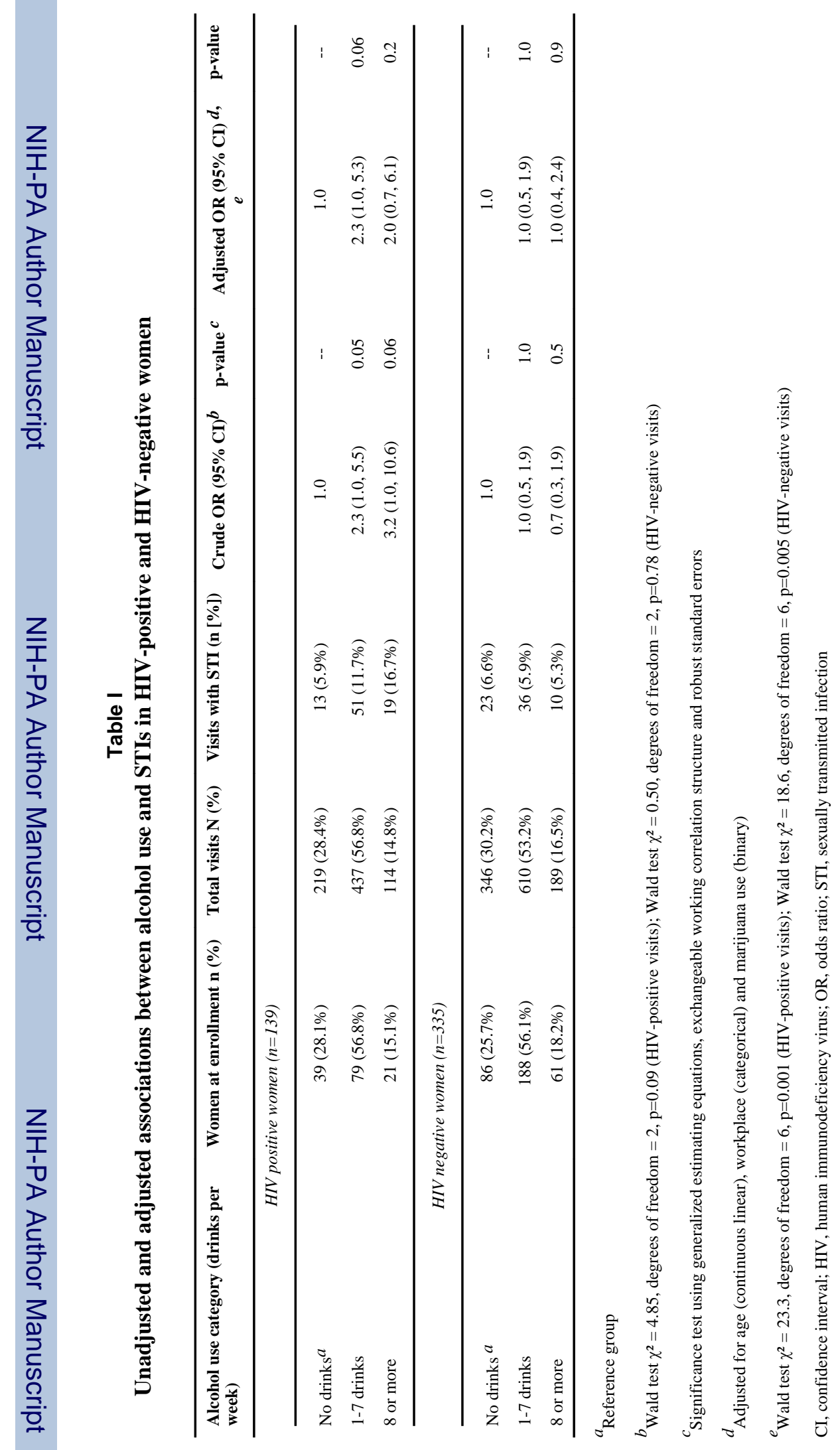

